\title{
Photoresponse and carrier transport of protocrystalline silicon multilayer films
}

\author{
YU Wei*, ZHU HaiRong, ZHAO Yi, SUN YuKai, LU HaiJiang \& FU GuangSheng \\ College of Physics Science and Technology, Hebei University, Baoding 071002, China
}

Received September 26, 2011; accepted February 17, 2012; published online May 6, 2012

\begin{abstract}
Alternating multilayer films of hydrogen diluted hydrogenated protocrystalline silicon (pc-Si:H) were prepared using a plasma-enhanced chemical vapor deposition technique. The microstructure of the deposited films and photoresponse characteristics of their Schottky diode structures were investigated by Raman scattering spectroscopy, Fourier transform infrared spectroscopy and photocurrent spectra. Microstructure and optical absorption analyses suggest that the prepared films were pc-Si:H multilayer films with a two-phase structure of silicon nanocrystals (NCs) and its amorphous counterpart and the band gap of the films showed a decreasing trend with increasing crystalline fraction. Photocurrent measurement revealed that silicon NCs facilitate the spatial separation of photo-generated carriers, effectively reduce the non-radiative recombination rate, and induce a photoresponse peak value shift towards the short-wavelength side with increasing crystallinity. However, the carrier traps near the surface defects of silicon NCs and their spatial carrier confinement result in a significant reduction of the diode photoresponse in the longwavelength region. An enhancement of the photoresponse from 350 to $1000 \mathrm{~nm}$ was observed when applying an increased bias voltage in the diode, showing a favorable carrier transport and an effective collection of photo-generated carriers was achieved. Both the spatial separation of the restricted electron-hole pairs in silicon NCs and the de-trapping of the carriers at their interface defects are responsible for the red-shift in photoresponse spectra and enhancement of external quantum efficiency. The results provide fundamental data for the carrier transport control of high-efficiency pc-Si:H solar cells.
\end{abstract}

pc-Si:H, photoresponse, carrier transport, crystalline fraction

Citation: $\quad$ Yu W, Zhu H R, Zhao Y, et al. Photoresponse and carrier transport of protocrystalline silicon multilayer films. Chin Sci Bull, 2012, 57: 2624-2630, doi: 10.1007/s11434-012-5190-3

The hydrogenated amorphous silicon $(\mathrm{a}-\mathrm{Si}: \mathrm{H})$ solar cell has become one of the main structures of industrial silicon-based thin-film solar cells because of its low-cost, light weight and low-temperature deposition [1]. However, lightinduced degradation, an intrinsic characteristic of these materials, has greatly limited their practical application in novel photovoltaic technology [2]. In recent years, hydrogen diluted protocrystalline silicon (pc-Si:H) which exists just before the amorphous-to-microcrystalline transition, has attracted great interest because of its low light-induced degradation [3]. Many investigations [4,5] reveal that silicon nanocrystals (NCs) present in pc-Si:H films spatially separate photo-generated carriers and suppress the photocreation

\footnotetext{
*Corresponding author (email: yuwei@ @bu.edu.cn)
}

of dangling bonds, which would cause the conversion efficiency of pc-Si:H solar cells to be stabilized rapidly and avoid further degeneration under prolonged light illumination. In addition, the optical band gap of pc-Si:H films is broadened because of the presence of silicon NCs $[4,6]$, which expands the utilization of the solar spectrum when they are used rather than a-Si:H as the top part of tandem solar cells.

Understanding the microstructure and optoelectronic properties of pc-Si:H films has become an active field in the design of silicon-based thin film photovoltaic devices. Results show that pc-Si:H films are composed of small amounts of silicon NCs embedded in an amorphous silicon matrix, because of their position at the transition state between amorphous and microcrystalline silicon. Recent in- 
vestigations have shown that an increase in hydrogen dilution ratio under certain conditions could promote the formation of silicon NCs, further inducing the disorder-toorder structural transition [7]. Because the band gap and band structures of silicon NCs and their amorphous counterparts differ [8], a grain boundary of several atom layer in thickness exists between nanocrystalline and amorphous regions. The carriers transport mechanism in pc-Si:H films is complex, as it is a composite material made up of a nanocrystalline phase, a grain boundary and an amorphous phase. For photovoltaic applications, it is believed that the larger cross-section of the favored carrier traps of isolated silicon NCs than those of the defects, reduces the nonradiative recombination of photo-generated carriers in the amorphous silicon matrix [1], which helps to improve the photoresponse and suppress light-induced degradation. However, increased carrier traps in the defects [9] and carrier recombination within silicon NCs with increased proportions of silicon NCs will significantly affect the photovoltaic properties of pc-Si:H films. Therefore, it is critical to control the structure of $\mathrm{pc}-\mathrm{Si}: \mathrm{H}$ films and understand the carriers transport process to develop their advantages in solar cells.

In this paper, pc-Si:H multilayer films with different crystalline fractions were prepared by plasma-enhanced chemical vapor deposition (PECVD) technique with hydrogen dilution varied between alternate layers. During film deposition, the preparation of silicon NCs on the surface of amorphous silicon was achieved by increasing the hydrogen dilution. The amorphous silicon sublayer provides a uniform initial growth condition for silicon NCs and thus favors the uniformity in their size and structure. Furthermore, the alternating amorphous silicon/nanocrystalline silicon structure was unsuspected to favor the control of carrier transport. Raman spectroscopy and Fourier transform infrared (FTIR) spectroscopy were used to analyze the microstructure and bonding characteristics. On this basis, a Schottky diode structure glass/ZnO:Al/i-pc-Si:H multilayer/Al was prepared. The effects of silicon NCs on photo-generated carrier transport and collection were discussed through comparison of the photoresponse of pc-Si:H multilayer films possing different crystalline fractions.

\section{Experimental}

In this work, pc-Si:H multilayer films were deposited by an inductively coupled PECVD technique, using $\mathrm{SiH}_{4}$ and $\mathrm{H}_{2}$ as reactants. The deposition pressure and radio frequency power were fixed at $0.3 \mathrm{~Pa}$ and $240 \mathrm{~W}$, respectively. The substrate temperature remained at $250^{\circ} \mathrm{C}$ during deposition. The flow rate of $\mathrm{SiH}_{4}$ was fixed at $0.6 \mathrm{sccm}$ (standard-state cubic centimeters per minute). The hydrogen flow rate was adjusted between 27 (a low $\mathrm{H}_{2}$-diluted sublayer) and 60 sccm (a highly $\mathrm{H}_{2}$-diluted sublayer), at which nearly a-Si:H and hydrogenated nanocrystalline silicon (nc-Si:H) films can be achieved, respectively. The growth period of the a-Si:H sublayer was fixed at 2 min (thickness $10 \mathrm{~nm}$ ), and that of the nc-Si:H sublayer was set at 2, 3, 4 and 5 min to modify the microstructure of the deposited films (M1, M2, M3 and M4, respectively). We adjusted the total cycles of $\mathrm{H}_{2} / \mathrm{SiH}_{4}$ to $16,13,10$ and 8 to maintain the thickness of our samples at around $300 \mathrm{~nm}$. Figure 1 shows the structure of the pc-Si:H multilayer films used to perform photoresponse characterization. The samples had the following Schottky diode structure: glass/ZnO:Al/i-pc-Si:H multilayer/Al. A single-layer a-Si:H film device with the same structure was prepared as a reference sample for comparison. Raman spectra were recorded using T64000 Raman (HORIBA Jobin Yvon Raman Division, Paris, French) system with excitation by an argon ion laser, operating at $532 \mathrm{~nm}$. The local bonding configurations of the films were characterized by infrared transmission measurement on a Bio-Rad $60 \mathrm{~V}$ FTIR (Bio-Rad Laboratories, Hercules, CA, USA) system. The photoresponse spectra and external quantum efficiency (EQE) were determined from spectral response measurements that were performed using a Solar Cell Scan 100 system that had a Tungsten lamp and a monochromator to produce mono-chromatic light, calibrated with a reference Si photodetector.

\section{Results and discussion}

\subsection{Raman analysis}

Raman spectroscopy as an effective tool has been widely used to estimate the characteristic size of crystallites and characterize their bonding structure in silicon-based thin films. Figure 2(a) displays the Raman spectra of the deposited films. Relative to a-Si:H films, a weak Raman scattering peak appeared in multilayer films at $515 \mathrm{~cm}^{-1}$, the intensity of which increased with increasing nc-Si:H sublayer growth period. The appearance of these subtle structures

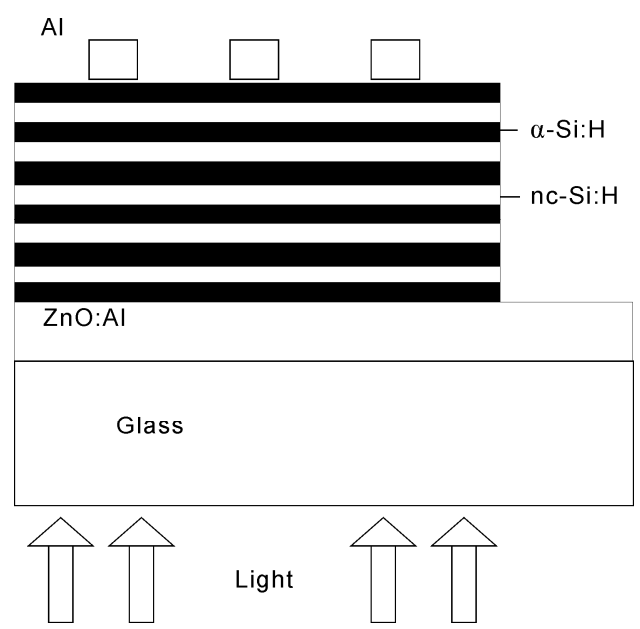

Figure 1 Structure of pc-Si:H multilayer films. 

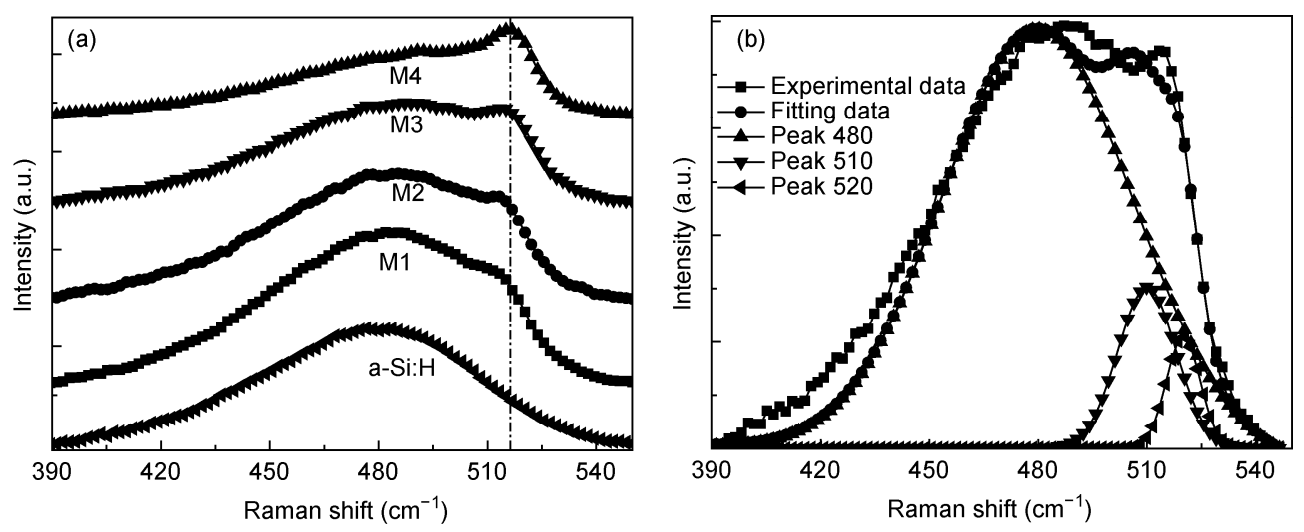

Figure 2 (a) Raman spectra of multilayer films M1, M2, M3 and M4 and a-Si:H single layer; (b) fitted spectra for multilayer films M3.

clearly indicates the presence of small crystallites with new structures in the sample. Compared with previous reports [10-12] on pc-Si:H films, the multilayer films prepared here possessed a high crystalline component.

Gaussian deconvolution of the Raman spectra was performed to obtain the crystalline volume fraction, $X_{\mathrm{c}}$, and grain size, and the results are shown in Figure 2(b). Three typical satellite components were obtained, corresponding to the crystalline Gaussian peaks, $I_{\mathrm{c}}\left(\sim 520 \mathrm{~cm}^{-1}\right)$, amorphous Gaussian peak, $I_{\mathrm{a}}\left(\sim 480 \mathrm{~cm}^{-1}\right)$, and intermediated Gaussian peak, $I_{\mathrm{m}}\left(\sim 510 \mathrm{~cm}^{-1}\right)$ [13], respectively. The crystalline fraction was calculated from

$$
X_{\mathrm{c}}=\left(I_{\mathrm{c}}+I_{\mathrm{m}}\right) /\left(I_{\mathrm{c}}+I_{\mathrm{m}}+I_{\mathrm{a}}\right),
$$

assuming "Raman crystallinity" as the ratio between areas corresponding to crystal and amorphous phase peaks. The crystallite size can be calculated from the formula:

$$
\Delta \omega(D)=-A(\alpha / D)^{\gamma},
$$

where $\Delta \omega(D)$ is the shift in the peak of the sample compared with that of crystalline silicon, $\alpha$ is the lattice constant of crystalline silicon, $A=47.41 \mathrm{~cm}^{-1}$, and $\gamma=1.44$ [14]. The parameters of the prepared films are shown in Table 1. As can be seen in the table, the crystalline fraction and grain size of multilayer films increased monotonically with increasing nc-Si:H sublayer growth period. When the growth period of the nc-Si:H sublayer increased from 2 to $4 \mathrm{~min}$, the crystal fraction of the multilayer films increased from $5 \%$ to $13 \%$. However, when the growth period increased to
5 min, the crystal fraction increased markedly to $39 \%$, indicating that the multilayer films had become nc-Si:H films. Thus, pc-Si:H multilayer films with highly crystalline fractions were prepared when the nc-Si:H sublayer growth periods were 2, 3 and $4 \mathrm{~min}$.

\section{$2.2 \quad$ FTIR analysis}

The chemical bonding structure and bonded hydrogen content of multilayer films can be obtained from FTIR spectra. Figure 3(a) presents the FTIR transmission spectra of multilayer films. Three peaks are discernible around 630, 880 and $2000 \mathrm{~cm}^{-1}$, which can be assigned to $\mathrm{Si}-\mathrm{H}$ waggling, $\mathrm{Si}-\mathrm{H}$ bending and $\mathrm{Si}-\mathrm{H}$ stretching vibration modes, respectively $[15,16]$. A shoulder peak emerges in the vicinity of $2100 \mathrm{~cm}^{-1}$ and the intensity increases with increasing nc-Si:H sublayer growth period. The absorption band in the wave number region $1900-2200 \mathrm{~cm}^{-1}$ can be decomposed into two Gaussian peaks, located at 2000 and $2080 \mathrm{~cm}^{-1}$ (Figure 3(b), inset). The former peak can be attributed to $\mathrm{Si}-\mathrm{H}$ bond vibration absorption, and the latter is derived from the stretching vibration mode of the silicon dihydrogen bond $\left(\mathrm{Si}-\mathrm{H}_{2}\right)$ groups or clustered $\mathrm{Si}-\mathrm{H}$ bonds in grain boundary regions [1]. The integrated intensity ratio was computed, and the results are shown in Figure 3(b). As can be seen, the integral intensity ratio of the $2080 \mathrm{~cm}^{-1}$ peak increases compared to that of the $2000 \mathrm{~cm}^{-1}$ peak with increasing nc-Si:H sublayer growth period. The $2080 \mathrm{~cm}^{-1}$ absorption peak of multilayer films can be assigned to clustered $\mathrm{Si}-\mathrm{H}$ bonds in the grain boundary regions, by taking

\begin{tabular}{|c|c|c|c|c|}
\hline Sample & Deposition time of nc-Si:H sublayer (min) & $X_{\mathrm{c}}(\%)$ & Mean crystallite size (nm) & Raman peak $\left(\mathrm{cm}^{-1}\right)$ \\
\hline $\mathrm{a}-\mathrm{Si}: \mathrm{H}$ & 0 & 0 & 0 & 480 \\
\hline M1 & 2 & 5 & 2.9 & 511.8 \\
\hline M2 & 3 & 8 & 3.0 & 512.5 \\
\hline M3 & 4 & 13 & 3.2 & 513.5 \\
\hline M4 & 5 & 39 & 4.2 & 516.5 \\
\hline
\end{tabular}

Table 1 Parameters extracted from the Raman analysis 

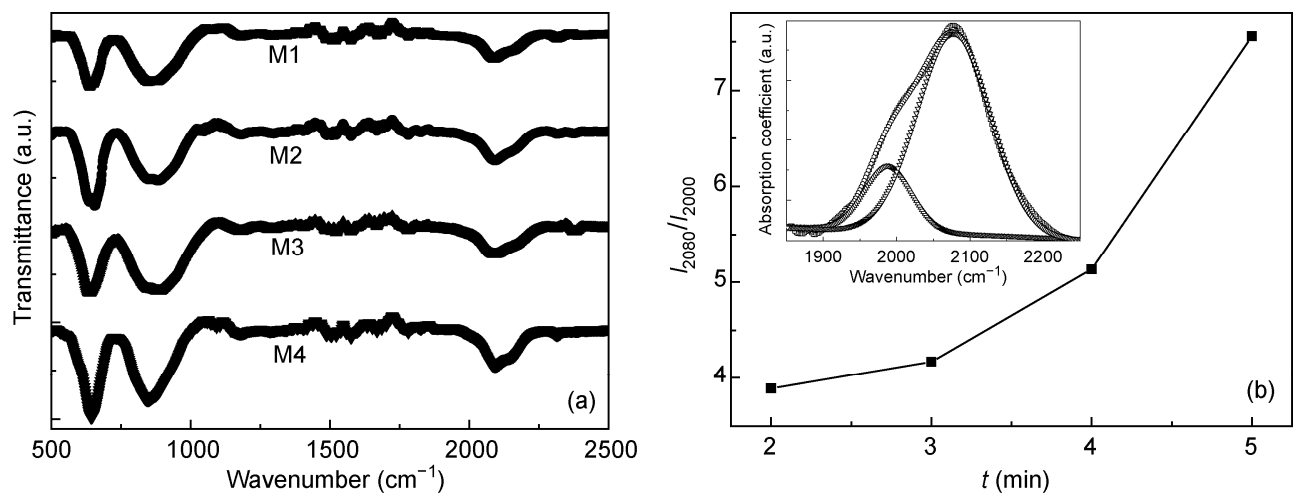

Figure 3 (a) FTIR transmission spectra of multilayer films M1, M2, M3 and M4; (b) integral intensity ratio of multilayer films M1, M2, M3 and M4 at peak $2100 \mathrm{~cm}^{-1}$. The inset shows the fitted spectra for M3.

into account the frequency shift and intensity enhancement near $630 \mathrm{~cm}^{-1}$ (Figure 3(a)). This conclusion is supported by the enlargement of the peak near $880 \mathrm{~cm}^{-1}$, corresponding to the vibrational mode of higher-order $\mathrm{Si}-\mathrm{H}$. This indicates that the bond density of $\mathrm{Si}-\mathrm{H}$ clusters at the surface of silicon NCs increases with the increasing crystallinity. Combined with the Raman analysis, the results reveal that the grain density and size of multilayer films increased with increasing nc-Si:H sublayer growth peroid, and that the clustered $\mathrm{Si}-\mathrm{H}$ bond density also increased, with the effect of terminating the nc-Si surface.

\subsection{Photoresponse}

UV-visible absorption spectra was used to analyze the optical band gap to explore the carrier transport properties of pc-Si:H multilayer films. Figure 4 shows the optical band gap $\left(E_{\mathrm{g}}\right)$ and the disorder factor $(B)$ of the deposited films. It can be seen in this figure that the optical band gap of pc-Si:H films increases significantly with the presence of silicon NCs. This is closely related to the microstructural change in pc-Si:H films: the emergence and growth of silicon NCs will inevitably reduce the overall disorder in multilayer films. The disorder factor in Figure 4 shows an increasing trend with increasing nc-Si:H sublayer growth period. As the microstructure disorder decreases, a narrow

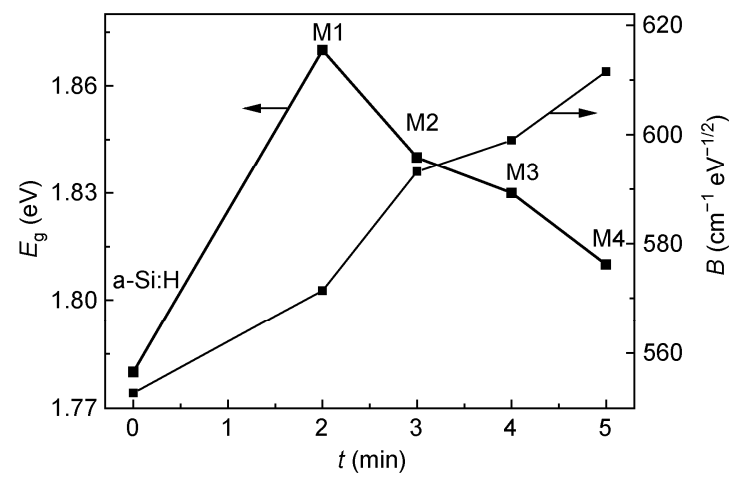

Figure 4 Band gap and $B$ factor of multilayer films M1, M2, M3 and M4 and a-Si:H single layer. band-tailed distribution will be formed, so the corresponding optical band gap increases compared to amorphous silicon. With an increasing nc-Si:H sublayer growth period, the crystalline fraction and the grain size of the deposited films increase monotonically, with a reduced proportion of amorphous silicon, so there is a downward trend in the band gap of pc-Si:H films.

The Schottky diodes structure of glass/ZnO:Al/i-pc-Si:H multilayer/Al were prepared and their photoresponses and EQEs determined. Figure 5 shows the corresponding spectral response and EQE of pc-Si:H multilayer and a-Si:H single layer film devices. Because the optical band gap of pc-Si:H multilayer films is relatively high compared to that of an a-Si:H single layer, as can be seen in Figure 4, the spectral response range of the corresponding optoelectronic device will move to the short-wavelength region. As Figure 5(a) shows, the M1 device exhibited a strong photoresponse signal and blue shift of the mid-peak relative to the a-Si:H device. This result is closely related to the presence of silicon NCs. Analysis of the microstructure reveals that the multilayer films were composed of isolated silicon NCs and an amorphous region. The absorption of short-wavelength photons in pc-Si:H films mainly occurs in the amorphous region because of its wider optical band gap and higher optical absorption intensity [4] and the existence of a higher proportion of amorphous region was considered. Taking into account the thicknesses of the a-Si:H $(\sim 10 \mathrm{~nm})$ and incubation layers (several $\mathrm{nm}$ ), the average distance between silicon NCs in multilayer films is much shorter than the carrier diffusion length in a-Si:H (>100 nm) [4]. Thus, the photo-generated carriers in the a-Si:H matrix of all sublayers can diffuse to the grain boundary regions surrounding the silicon NCs. The carrier trapping of the crystallites is therefore dominated by diffusion and the carrier lifetime is inversely proportional to the capture radius, $d$. The silicon NCs have a much larger capture radius (corresponding to their grain size) than that of the dangling-bond defects $(d \approx$ several $\AA$ ) [17]. Therefore, silicon NCs favor photo-generated carrier traps and hence suppress the photocreation of dangling-bonds, which is usually attributed to the nonradia- 
tive recombination in a-Si:H matrix. Additionally, the energy band near the grain boundary is concave-downward, because of the carriers trapped at the interface defects of the a-Si:H sublayer [8]. Therefore, the photo-generated carriers can easily be separated spatially and the probability of recombination reduced because of the presence of silicon NCs and, consequently, a strong photoresponse signal is obtained in pc-Si:H films. However, as can be seen in Figure 5(a), the photoresponse signal of the M1 device was small compared to that of a-Si:H in the long-wavelength region. This reflects the impedimental effect of the grain boundary. The carriers generated by low-energy photons are hindered at silicon NCs and interface defects because of the smaller band gap of nc-Si:H relative to a-Si:H. Therefore, the photoresponse of pc-Si:H films is markedly reduced in the long-wavelength range. This can be seen clearly in the EQE curves shown in Figure 5(b). Relative to a-Si:H, M1 showed a high EQE in the short-wavelength region but only a weak $\mathrm{EQE}$ in the long-wavelength region.

It can also be seen that the photoresponse signals and EQEs of M1, M2 and M3 showed a decreasing trend revealing defect traps for carriers at the silicon NCs/a-Si interface. The preceding analyses of Raman and FTIR spectra show that the crystal fraction and grain size are on the rise with increasing nc-Si:H sublayer growth period, i.e. the silicon NCs density increases in multilayer films. Nanosized silicon grains embedded in pc-Si:H multilayers suppress the photocreation of dangling bonds in a-Si matrix acting as the radiative recombination centre of photogenerated carriers [5]. Thus, the increasing density of silicon NCs will increase the probability of carrier recombination. When the silicon NCs density and size increase, defects in the grain boundary will also increase [9]. As a nonradiative recombination center, interfacial defects would increase the rate of photo-generated carrier non-radiative recombination, resulting in lower photoresponse signals and EQEs. Additionally, the thickness of a-Si:H gradually decreased with increasing nc-Si:H sublayer growth period. The reduced proportion of light absorption in a-Si:H would also influence the overall photoresponse signal. However, when the nc-Si:H sublayer growth time increased to $5 \mathrm{~min}$, the photoresponse signal of sample M4 showed a rising trend and peak shifts towards the long-wavelength range. As can be seen in the Raman spectra, the crystal fraction of sample M4 was 39\%, so a nc-Si:H film had been formed. In this case, the transport of photo-generated carriers would be achieved by tunneling among silicon NCs. Therefore, a strong photoresponse signal can be obtained by reducing the interface traps.

\subsection{Bias effect in photoresponse}

The above analysis of EQE shows that there is a limited collection of photo-generated carriers in $\mathrm{pc}-\mathrm{Si}: \mathrm{H}$ multilayer films. According to the three-phase model [1,4], pc-Si:H films can be regarded as a composite of silicon NCs a few nanometers in size, a grain boundary and a-Si. As silicon $\mathrm{NCs}$ and a-Si:H have very different band gap and band structures, heterojunction structures will be formed in the grain boundary. The nc-Si:H sublayer behaviors as a well layer, while the a-Si:H sublayer functions as a barrier layer. Considerable numbers of carriers will accumulate in the quantum well $(\mathrm{QW})$ region because of the restriction of carrier transport there. Additionally, interface traps for carriers will occur at the $\mathrm{nc}-\mathrm{Si} / \mathrm{a}-\mathrm{Si}$ interface. Under applied bias, the energy band of QWs will be tilted and the restriction and trap effect on carriers will be significantly reduced, which will help carrier transport and collection. Figure 6 shows the photoresponse and EQE of sample M3 under a series of applied bias voltages. As expected, the carrier collection was improved when bias was applied, indicating that the recombination/trap of photo-generated carriers was substantial depressed. As can be seen in Figure 6(a), the photoresponse signal at long-wavelength sharply increased with applied bias. The bias boosting of the photoresponse reach more than 2 orders of magnitude when the wavelength was between 650 and $800 \mathrm{~nm}$. To analyze the mechanism of carrier transport, the photoresponse gain, $G=\left(I_{\mathrm{v}}-\right.$ $\left.I_{0}\right) / I_{0}$, as a function of incident wavelength is shown in the inset of Figure 6(a), where $I_{\mathrm{v}}$ and $I_{0}$ are the photoresponse

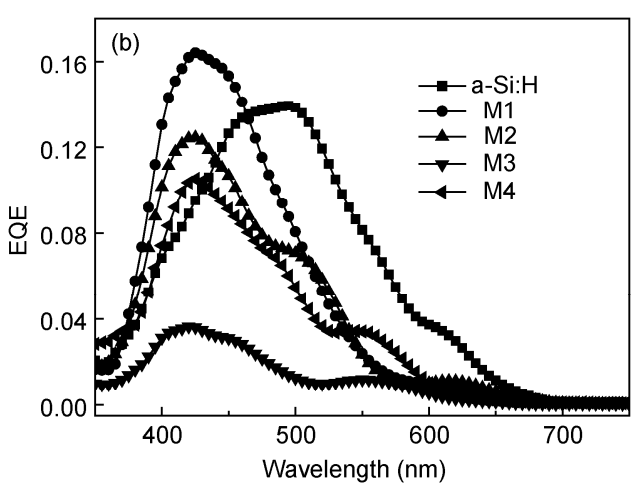

Figure 5 (a) Photoresponse spectral of multilayer films M1, M2, M3 and M4 and a-Si:H single layer; (b) EQE of multilayer films M1, M2, M3 and M4 and a-Si:H single layer. 

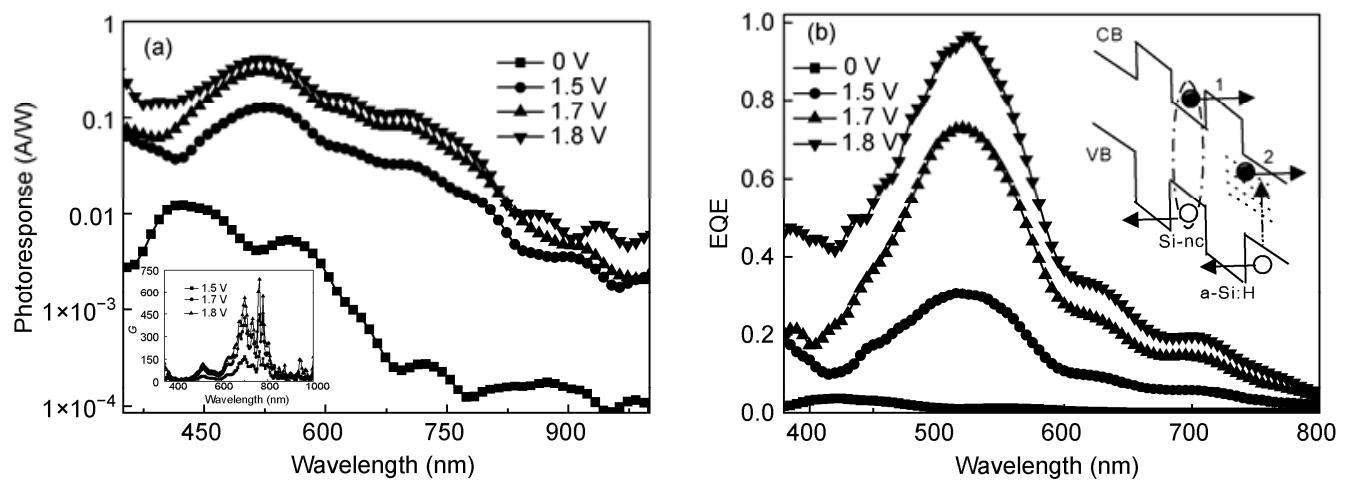

Figure 6 (a) Photoresponse spectra (logarithmic scale) of pc-Si:H multilayer films M3 under applied bias voltages. The inset shows photoresponse gains at different biases. (b) EQE of pc-Si:H multilayer film M3 under applied bias voltages. The inset illustrates the carrier transport processes of pc-Si:H multilayer films under bias.

values under various applied biases and zero bias, respectively. Three distinct peaks at about 770, 700 and $515 \mathrm{~nm}$ are presented in the $\mathrm{G}$ curves. As shown in Figure 4, the optical band gap of sample M3 was about $1.83 \mathrm{eV}$. Considering that the band gap of $\mathrm{Si} \mathrm{NCs}$ is smaller than that of pc-Si films, the gain peak at about $700 \mathrm{~nm}$ reflects increased spatial separation of photo-generated carriers in silicon NCs. Unlike the a-Si material, the excitation state of the bounded electron-hole pair in silicon NCs can be formed by the absorption of a photon [18], and easily separate to form a free-photogenerated carrier under an electric field (process 1 in the inset of Figure 6(b)).

The gain peak at about $770 \mathrm{~nm}$ can be ascribed to the increase in the photoresponse related to grain boundary defects. It has been reported that a defect level is located just $0.28 \mathrm{eV}$ below the conduction band of silicon NCs at their interface with amorphous parts because of bond distortions [19]. Under subband gap illumination, electrons from the silicon NCs valence band are photoexcited to the interface states, where they become trapped. Under bias voltages, the tunneling of trapped electrons from the trap level to the conduction band of silicon NCs is possible and they will thus contribute to carrier transport, resulting in the enhancement of the photoresponse signal. Therefore, the gain peak at $770 \mathrm{~nm}$ indicates that the photo-generated carriers contained in these defect states are excited to become free carriers under an applied electric field (process 2 in the inset of Figure 6(b)).

In addition to 700 and $770 \mathrm{~nm}$ gain peaks, a weak gain peak at $515 \mathrm{~nm}$ appeared. This means that the applied bias also has an effect on the photo-generated carrier transport which is larger than the band gap. As can be seen in the inset of Figure 6(a), the gain value at various bias voltages is relatively weak at the $515 \mathrm{~nm}$ peak compared to those at 700 and $770 \mathrm{~nm}$. This result can be understood by the effective collection of photo-generated carriers even in the absence of an applied bias because the energy of the carriers at this level exceeds the band bank of this film. From the above analyses, the enhancement of the photoresponse sig- nal in pc-Si:H films under applied electric fields can be understood as a joint effect of the spatial separation of the bounded electron-hole pairs in silicon NCs and the de-trapping of the carriers at the interface defects.

Silicon NCs surface defects play an important role on the photoelectric conversion in $\mathrm{pc}-\mathrm{Si}: \mathrm{H}$. The photoresponse signal increased significantly when the photo-energy was less than the band gap of silicon NCs under bias voltages because of trap-assisted light absorption at the silicon NCs interface. As can be seen in Figure 6(b), the M3 device spectral response range with high EQE was extended to 800 $\mathrm{nm}$. For pc-Si:H solar cells, an enhancement of photoelectric conversion efficiency can be obtained in a specific spectral range by adjusting the interface states density distribution. On the other hand, silicon NCs interface levels have a significant influence on the carrier transport excited by short-wavelength photons. First, the traps at a-Si/nc-Si interface defects will result in a reduction in the photoresponse signal. The de-trapping of restricted carriers will then contribute to the enhancement of photoresponse signal under bias (as shown in Figure 6(a)). Second, the electric field direction generated by the trapped carriers is opposite to the external electric field. Therefore, the de-trapping of restricted carriers will increase the tilting of the energy level for the multilayer structure and increase the carrier transfer rate. Figure 6(b) shows that the EQE mid-peak of the M3 device shifts toward the long-wavelength region with increasing applied bias. Therefore, the carriers generated by short-wavelength photon in a pc-Si:H solar cell can be effectively collected through control of the carrier trap process on defect states by applying an appropriate bias voltage.

\section{Conclusions}

Multilayer pc-Si:H films with a tunable microstructure and optical band gap were successful deposited through adjusting the growth period of the nc-Si:H sublayer. Raman spec- 
troscopy and FTIR spectroscopy analyses show that the prepared pc-Si:H films possessed a two-phase structure of isolated silicon NCs and an amorphous silicon matrix. The photoresponses of multilayer film Schottky diodes in the presence and absence of an applied bias were analyzed. Because isolated silicon NCs have a much larger capture cross-section than that of amorphous silicon interface defects, they can rapidly capture and spatially separate photo-generated carriers. This can suppress nonradiative recombination, leading to a high collection efficiency of carriers in the short-wavelength region. However, the carriers generated by long-wavelength photons are hindered because of the higher barrier of the a-Si:H grain boundary, resulting in a reduction in the photoresponse signal. Applied bias voltages are believed to boost the spatial separation of the photo-generated carriers, enabling effective collection of the carriers restricted in silicon NCs and the grain boundary defects. A significant enhancement in the photoresponse and EQE of the diode was observed. In summary, the photoresponse and photo-generated carriers transport characteristics in pc-Si:H films were investigated. The introduction of silicon NCs was shown to significantly improve the performance of a-Si:H thin-film solar cells, thereby offering a means to tune the material's absorption and enable improved solar spectrum utilization. Thus, the results are expected to serve as a useful reference for the practical fabrication of new, high-efficiency solar cells.

This work was supported by the National Natural Science Foundation of China (60878040 and 60940020).

1 Lim K S, Kwak J H, Kwon S W, et al. Highly and rapidly stabilized protocrystalline silicon multilayer solar cells. Mater Res Soc Symp Proc, 2005, 862: A11.2.1-A11.2.12

2 Staebler D L, Wronski C R. Reversible conductivity changes in discharge-produced amorphous Si. Appl Phys Lett, 1997, 31: 292-294

3 Kwon S W, Kwak J, Myong S Y, et al. Characterization of the protocrystalline silicon multilayer. J Non-Crystal Solids, 2006, 352: 1134-1137

4 Myong S Y, Kwon1 S W, Kondo M, et al. Development of a rapidly stabilized protocrystalline silicon multilayer solar cell. Semicond Sci
Technol, 2006, 21: L11-L15

5 Myong S Y, Kwon S W, Lim K S, et al. Inclusion of nanosized silicon grains in hydrogenated protocrystalline silicon multilayers and its relation to stability. Appl Phys Lett, 2006, 88: 083118

6 Yoon J H, Lee C H. Stability of undoped hydrogenated amorphous silicon multilayer film grown with alternating substrate temperature. Appl Phys Lett, 1996, 69: 1250-1252

7 Zhang R, Chen X Y, Zhang K, et al. Photocurrent response of hydrogenated nanocrystalline silicon thin films. J Appl Phys, 2006, 100: 104310

8 Gao X Y, Zhao J T, Liu Y F, et al. Characterized microstructure and electrical properties of hydrogenated nanocrystalline silicon films by Raman and electrical conductivity spectra. Acta Phys Polon A, 2009, 115: 738-741

9 Han D X, Wang K D, Owens J M, et al. Hydrogen structures and the optoelectronic properties in transition films from amorphous to microcrystalline silicon prepared by hot-wire chemical vapor deposition. J Appl Phys, 2003, 93: 3776-3783

10 Ahn J Y, Jun K H, Lim K S, et al. Stable protocrystalline silicon and unstable microcrystalline silicon at the onset of a microcrystalline regime. Appl Phys Lett, 2003, 82: 1718-1720

11 Zhang S B, Liao X B, Xu Y Y, et al. Microstructure of the silicon film prepared near the phase transition regime from amorphous to nanocrystalline. Mat Res Soc Symp Proc, 2003, 737: F8.11.1F8.11.7

12 Wu X L, Tong S, Liu X N, et al. X-ray diffraction study of alternating nanocrystalline silicon/amorphous silicon multilayers. Appl Phys Lett, 1997, 70: 838-840

13 Gracin D, Sancho-Paramon J, Juraic K, et al. Analysis of amorphousnano-crystalline multilayer structures by optical, photo-deflection and photo-current spectroscopy. Micron, 2009, 40: 56-60

14 Viera G, Huet S, Boufendi L. Crystal size and temperature measurements in nanostructured silicon using Raman spectroscopy. J Appl Phys, 2001, 90: 4175-4183

15 Jun K H, Ouwens J D, Schropp R E I. Low degradation and fast annealing effects of amorphous silicon multilayer processed through alternate hydrogen dilution. J Appl Phys, 2000, 88: 4881-4888

16 Han D X, Wang K D, Owens J M, et al. Hydrogen structures and the optoelectronic properties in transition films from amorphous to microcrystalline silicon prepared by hot-wire chemical vapor deposition. J Appl Phys, 2003, 93: 3776-3783

17 Kamei T, Stradins P, Matsuda A. Effects of embedded crystallites in amorphous silicon on light-induced defect creation. Appl Phys Lett, 1999, 74: 1707-1709

18 Zhang R, Chen X Y, Lu J J, et al. Photocurrent of hydrogenated nanocrystalline silicon thin film/crystalline silicon heterostructure. J Appl Phys, 2007, 102: 123708

19 He Y L, Hu G Y, Yu M B, et al. Conduction mechanism of hydrogenated nanocrystalline silicon films. Phys Rev B, 1999, 59: 1535215357

Open Access This article is distributed under the terms of the Creative Commons Attribution License which permits any use, distribution, and reproduction in any medium, provided the original author(s) and source are credited. 